ARTICLE

\title{
Specific features and assembly of the plant mitochondrial complex I revealed by cryo-EM
}

Heddy Soufari ${ }^{1}$, Camila Parrot ${ }^{1}$, Lauriane Kuhn (i) ${ }^{2}$, Florent Waltz (i) ${ }^{1 凶} \&$ Yaser Hashem (D) ${ }^{1 凶}$

Mitochondria are the powerhouses of eukaryotic cells and the site of essential metabolic reactions. Complex I or NADH:ubiquinone oxidoreductase is the main entry site for electrons into the mitochondrial respiratory chain and constitutes the largest of the respiratory complexes. Its structure and composition vary across eukaryote species. However, high resolution structures are available only for one group of eukaryotes, opisthokonts. In plants, only biochemical studies were carried out, already hinting at the peculiar composition of complex I in the green lineage. Here, we report several cryo-electron microscopy structures of the plant mitochondrial complex I. We describe the structure and composition of the plant respiratory complex I, including the ancestral mitochondrial domain composed of the carbonic anhydrase. We show that the carbonic anhydrase is a heterotrimeric complex with only one conserved active site. This domain is crucial for the overall stability of complex I as well as a peculiar lipid complex composed of cardiolipin and phosphatidylinositols. Moreover, we also describe the structure of one of the plant-specific complex I assembly intermediates, lacking the whole $P_{D}$ module, in presence of the maturation factor GLDH. GLDH prevents the binding of the plant specific $P 1$ protein, responsible for the linkage of the $P_{P}$ to the $P_{D}$ module.

\footnotetext{
${ }^{1}$ Institut Européen de Chimie et Biologie, U1212 Inserm, Université de Bordeaux, 2 rue R. Escarpit, F-33600 Pessac, France. ${ }^{2}$ Plateforme protéomique Strasbourg Esplanade FRC1589 du CNRS, Université de Strasbourg, Strasbourg, France. ${ }^{凶}$ email: florent.waltz@etu.unistra.fr; yaser.hashem@inserm.fr
} 
C omplex I is the largest multimeric enzyme of the respiratory chain, composed of more than 40 protein subunits ${ }^{1}$. Fourteen are strictly conserved proteins subunits, vestige of complex I of bacterial origin ${ }^{2}$. The additional subunits, referred to as supernumerary subunits, were acquired during eukaryotes evolution $^{3}$. Part of these additional subunits are conserved among other eukaryotes and play essential roles for the structure, function and the association with the other respiratory chain complexes, e.g., to assemble into respirasome in animals ${ }^{4}$. Recently, several high resolution $3 \mathrm{D}$ structures of the complete mitochondrial complex I of opisthokonts were determined by cryo-EM in mammalian species $^{5-7}$ and in the aerobic yeast Yarrowia lipolytica ${ }^{8,9}$, revealing the organization of their additional specie-specific subunits. However, in plants, even though extensive biochemical characterization was conducted $^{10,11}$, high-resolution structures of mitochondrial complex I are yet to be derived. Early negative staining studies ${ }^{11}$ revealed the presence of a large additional membrane attached domain, absent from animal and yeast species, hinting at the peculiar structure and composition of the plant complex I.

In order to obtain a high-resolution structure of the plant mitochondrial complex I and its additional subunits, we purified mitochondria from Brassica oleracea var. botrytis, a close relative to the model plant Arabidopsis (both belong to the group of Brassicaceae plants), as previously described" (see "Methods" section). We recorded cryo-EM images of membrane complexes purified from sucrose gradient (see "Methods" section), corresponding to mitochondrial complex I. After particle sorting (see "Methods" section) we obtained cryo-EM reconstructions of two main complex I states: the full plant mitochondrial complex I, as well as reconstruction of a complex I assembly intermediate, without the $\mathrm{P}_{\mathrm{D}}$ module, and with the plant-specific assembly factor GLDH $^{13}$ (Supplementary Fig. 1). Focused refinement and particle polishing were performed in RELION3 ${ }^{14}$ (see "Methods" section), allowing reconstruction of the full complex I at $3.7 \AA$ resolution, focused classification on the membrane arm and $\mathrm{P}_{\mathrm{P}}$ module yielding $3.4 \AA$. The $\mathrm{P}_{\mathrm{D}}$ module being more flexible produced more scant densities reporting lower resolutions (Supplementary Figs. 1, 2). The cryo-EM maps allowed the determination of many well-resolved features and clear side-chain densities (Supplementary Fig. 3) that enabled modeling of the 45 proteins of the plant mitochondrial complex I (Supplementary Table 1), as well as several characteristic ligands that were directly identified from the density: the eight canonical FeS clusters of the matrix arm, the FMN of the $51 \mathrm{kDa}$ subunit, the NADPH molecule in the $39 \mathrm{kDa}$ subunit, three cardiolipins two phosphatidylinositol and one phosphatidylethanolamine (Supplementary Fig. 3). Ubiquinone was also clearly visible in the Q module (Supplementary Fig. 4).

\section{Results and discussion}

General description. The plant mitochondrial complex I has the classical open L-shape formed by the matrix and membrane arms (Fig. 1). In the matrix arm, electrons are transferred from $\mathrm{NADH}$ to ubiquinone along the FeS clusters with distances similar to what has been described in bacteria and opisthokonts mitochondrial complex I (Supplementary Fig. 5). The membrane part, composed of the proximal $\left(\mathrm{P}_{\mathrm{P}}\right)$ and distal $\left(\mathrm{P}_{\mathrm{D}}\right)$ modules relative to the matrix arm, where proton pumping takes place, is also conserved. Those highly conserved features are part of the 14 minimal protein subunits conserved in bacteria and other mitochondrial complex $\mathrm{I}^{1}$. The additional mitochondria specific proteins enhance the volume and mass of the complex I compared to bacteria. In plant, 25 proteins shared with aerobic yeast or mammals are present. Similarly to the previously obtained complex I structure $5,7,9,15$, the supernumerary subunits, specific to mitochondrial complex I, form a shell around the core subunits, adding nearly $400 \mathrm{kDa}$ of proteins to the conserved subunits (Supplementary Fig. 5). These supernumerary subunits are mainly composed of $\alpha$-helices and are largely interconnected with the core subunits. In the $\mathrm{Q}$ module of the matrix arm, our reconstructions allowed us to clearly visualize ubiquinone in the pocket formed by Nad1, PSST and Nad7 (Supplementary Fig. 4). Our results confirm the recently observed ubiquinone position described for Y. lipolytica ${ }^{8}$. During 3D classification of the mature complex, only one major conformation was observed. This would indicate that the plant complex I would be similar to the yeast complex I, where similar conformations are observed in both active and deactive states ${ }^{9}$, as opposed to mammalian complexes, where movement of the matrix arm relative to the membrane arm is observed between the states ${ }^{5}$.

Carbonic anhydrase: an ancestral component of mitochondrial complex I. The main feature of the plant complex I is the presence of an additional globular matrix-exposed domain, bound to the membrane arm. It holds an overlapping position with the NDUFA10/42-kDa subunit found in mammalian complex $\mathrm{I}^{5}$. This domain is formed by a trimeric complex, the carbonic anhydrase (CA). CA are zinc-containing enzymes that catalyze the reversible hydration of $\mathrm{CO}_{2}$ to $\mathrm{HCO}_{3}^{-}$, whose roles are usually carbon fixation and $\mathrm{pH}$ maintenance ${ }^{16}$. The carbonic anhydrase activity evolved independently several times during evolution, resulting in several CA enzyme families. The plant mitochondrial $\mathrm{CA}$ is part of the gamma-carbonic anhydrase $(\gamma \mathrm{CA})$ family, which was originally identified in the archaeon Methanosarcina thermophila ${ }^{17,18}$. Here, the overall structure of the $\gamma \mathrm{CA}$ is conserved: it is a trimer composed of characteristic left-handed beta-helix monomers, forming triangular prisms (Fig. 2a). However, in contrast to the archaeal enzyme, the $\gamma \mathrm{CA}$ present in plant complex I is an heterotrimer ${ }^{19}$. Indeed, in Arabidopsis five $\gamma$ CA genes were identified. Three have highly conserved catalytic domains and are termed $\gamma \mathrm{CA} 1-3$, similar to the archaeal enzyme, while the two other show less homology to their prokaryotic homologs especially in the catalytic domain and were thus termed $\gamma$ CA-like proteins ${ }^{20,21}$. Our reconstruction shows that the $\gamma \mathrm{CA}$ domain is formed by one copy of $\gamma \mathrm{CAL}$, built as $\gamma$ CAL1 representing the most proximal subunit to the membrane part and two copies of $\gamma \mathrm{CA}$. For the two $\gamma \mathrm{CA}$ subunits, one was clearly identified as $\gamma \mathrm{CA} 1$ but the scant density on the second, which could be explained by compositional heterogeneity, did not allow to clearly distinguish between $\gamma \mathrm{CA} 1$ and $\gamma \mathrm{CA} 2$, and was therefore built as $\gamma \mathrm{CA} 1$ (Fig. 2a and Supplementary Fig. 6). Each subunits have long $\mathrm{N}$ and C-terminal extensions interconnecting the 3 subunits and contacting the membrane subunits of complex I (Fig. 2c). These extensions also serve as anchors for the $\gamma \mathrm{CA}$ domain by interacting with the plant specific P2 protein $\mathrm{N}$ terminal part which is anchored in the membrane. The Cterminal part of the canonical Nad6 protein also contacts the CA domain stabilizing it. $\gamma \mathrm{CA}$ is an enzyme, where one histidine of one subunit and two histidine of the adjacent subunit contribute to coordinate a zinc atom. However, as $\gamma \mathrm{CAL}$ proteins lack two of those three histidines, it impairs the formation of the zinc coordination with both $\gamma \mathrm{CA}$ subunits. Hence the only conserved active site is at the interface of the two $\gamma$ CAs, which we confirmed by visualizing both the zinc and an additional density most likely corresponding to $\mathrm{HCO}_{3}{ }^{-}$(Fig. 2b and Supplementary Fig. 6). This strongly suggests that the $\gamma \mathrm{CA}$ domain associated with complex $\mathrm{I}$ is indeed active, even if a role in $\mathrm{HCO}_{3}{ }^{-}$fixation and/ or transport cannot be excluded, similarly to what is observed in cyanobacterial $\mathrm{CcmM}^{22}$. However, the function of complex Iassociated $\gamma \mathrm{CA}$ is not well understood. It was suggested to play a 
a

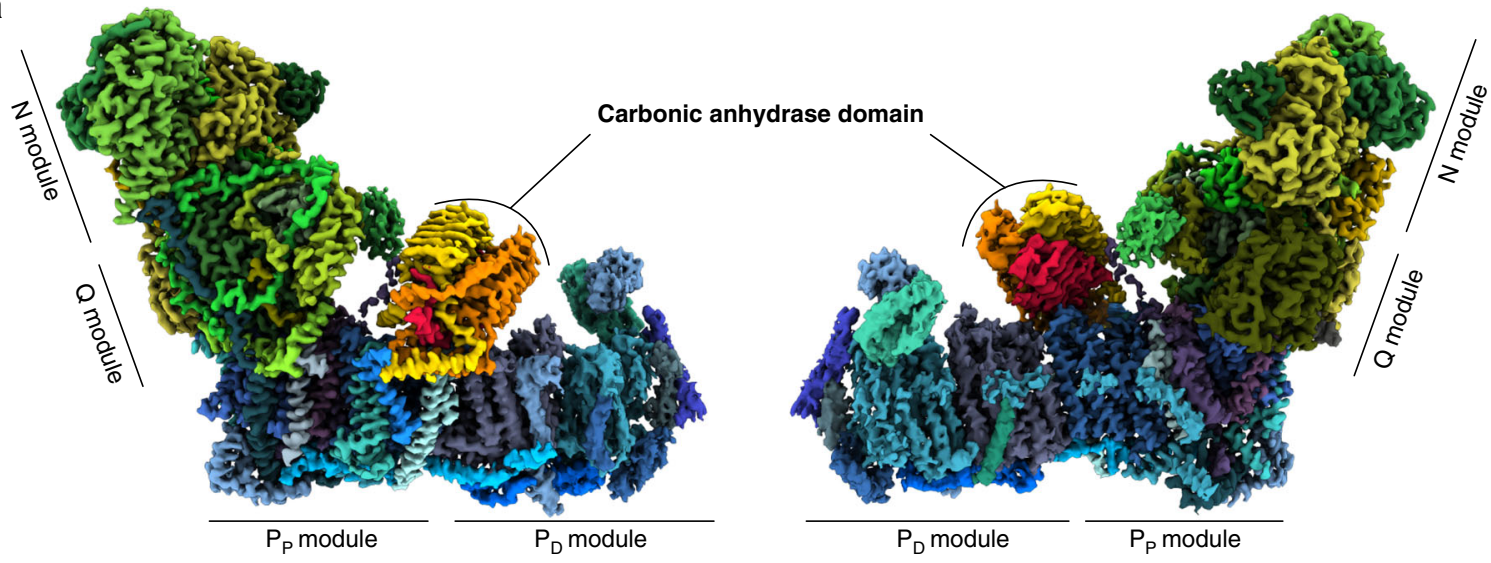

b

b $51 \mathrm{kDa} \quad 18 \mathrm{kDa}$

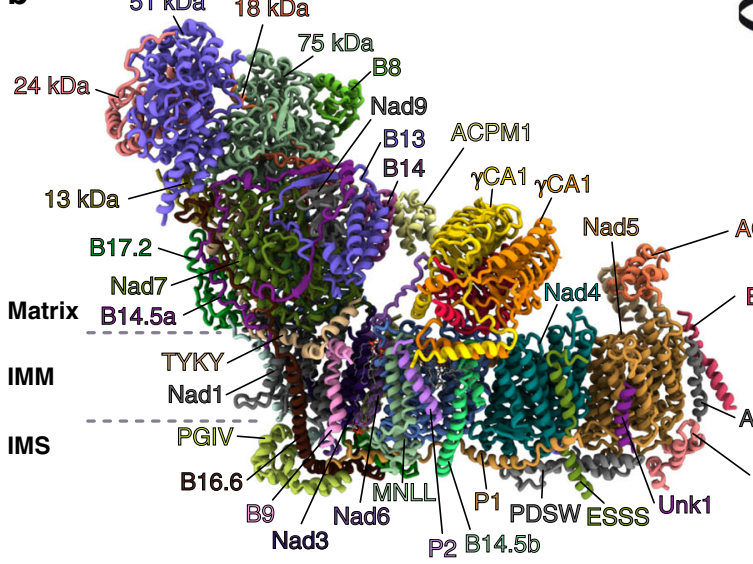
$\Rightarrow 180^{\circ}$

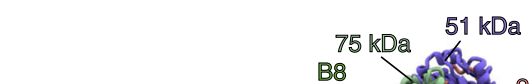

$51 \mathrm{kDa}$
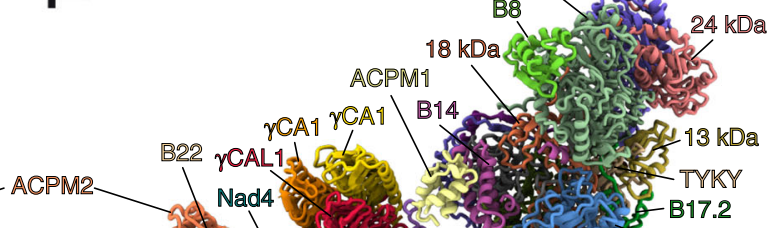

B12.1

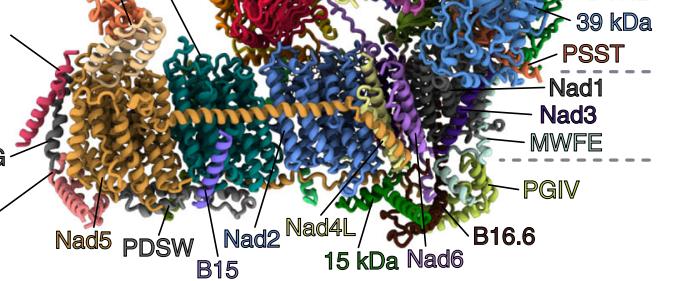

Fig. 1 Overall structure of the plant mitochondrial complex I. a Composite cryo-EM map of the complete plant complex I. The membrane part is displayed in blue shades, the matrix arm is displayed in green shades and the $\gamma$-carbonic anhydrase module is displayed in orange shades. Individual $N, Q_{1}, P_{D}$, and $P_{P}$ modules are indicated. $\mathbf{b}$ The resulting atomic model, each individual 45 proteins are annotated and displayed with a different color. IMM stands for inner mitochondrial membrane, IMS inter-membrane space.

role in complex I assembly as $\gamma \mathrm{CA}$ is found in early assembly intermediates of the complex and is essential for complex I formation $^{23}$. Indeed, embryo development of $\gamma \mathrm{CA}$ mutants is strongly delayed and seed development arrested before maturation. Moreover complemented mutants with inactive $\gamma \mathrm{CA}$ variants is sufficient to complement the mutant phenotype, showing that carbonic anhydrase activity is not essential ${ }^{20}$. Recently it was shown that carbonic anhydrase activity is also present in photosynthetic cyanobacterial complex I, but is carried out by a different type of CA enzyme, positioned at the end of the $\mathrm{P}_{\mathrm{D}}$ module that would contribute to proton generation ${ }^{24}$. However, in plant mitochondrial complex I, the only conserved active site is positioned at the most distal position relative to the membrane. Nevertheless, one cannot exclude its contribution to proton generation, although its effect would be relatively modest.

Interestingly, the CA domain is in tight interaction with a peculiar lipid complex positioned below the latter and between the plant specific $\mathrm{P} 2$ and proteins $\mathrm{B} 14.5 \mathrm{~b}$ and $\mathrm{Nad} 2$, close to the Nad2-Nad4 junction (Fig. 2d). This lipid complex is composed of one cardiolipin coordinated by two phosphatidylinositol. Each inositol heads sandwich the glycerol linker of the cardiolipin and stabilize it. This lipid block was also observed in the assembly intermediate (see below) and appears to be very stable. Given the essential role of lipids for complex I activity, this lipid block might be crucial for the plant complex I efficiency and stability, which might be why CA mutants are so heavily affected ${ }^{8,25}$. Altogether, our analysis strongly suggest that the $\gamma \mathrm{CA}$ is essential for the membrane part formation and stability, acting as an essential architectural factor that would remain even in mature complex.

Assembly intermediate of the plant complex I. During 3D classification, two classes of complex I lacking the most distal proton pumping components of the membrane domain-Nad4 and Nad5 and their associated additional protein subunits-were found to naturally accumulate in our sample (Supplementary Fig. 1). One of the two classes presented an additional globular density on the intermembrane space exposed side of complex I. Such density wasn't observed in full complex I classes. This class was thus attributed to an assembly intermediate of complex I (Fig. 3). In plants, such complex was already described to accumulate naturally. Indeed, extensive biochemical analyses identified complex $\mathrm{I}^{*}$ as an assembly intermediate lacking the most distal membrane components and presenting an additional factor, GLDH (L-Galactono-1,4-lactone dehydrogenase). GLDH was identified in our mass spectrometry data (Supplementary Table 1), along with the other components of complex I. Likewise $\gamma \mathrm{CA}, \mathrm{GLDH}$ is an enzyme catalysing the last enzymatic step of the ascorbate biosynthetic pathway in plants ${ }^{26}$. It was never found in the full complex I, however, it was shown to associate with complex I intermediates, complex $I^{*}$ being the largest, as well as with smaller complex intermediates that were not observed here ${ }^{23}$. Moreover, in gldh knock-out mutant, complex I 
a

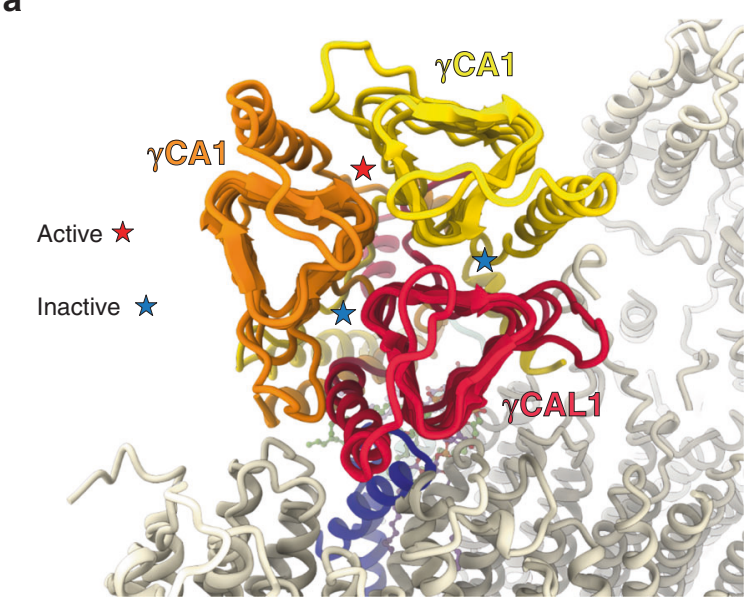

b

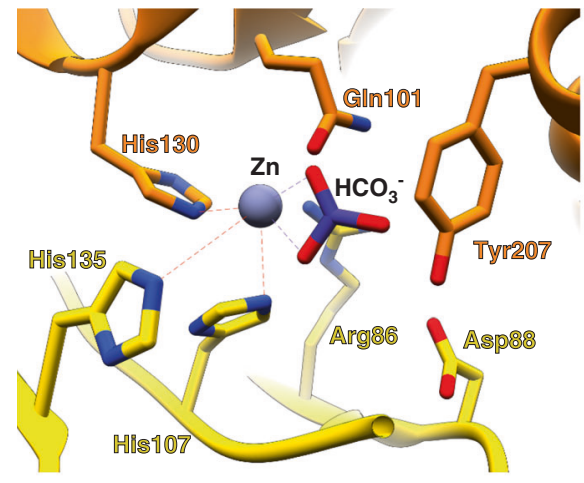

107

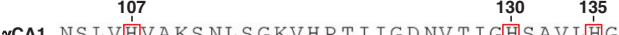

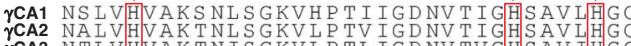
YCA3 NTLVHVAKTNISGKVLPTLIGDNVTVGHSAVIHG

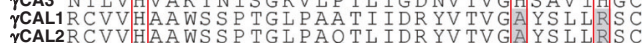

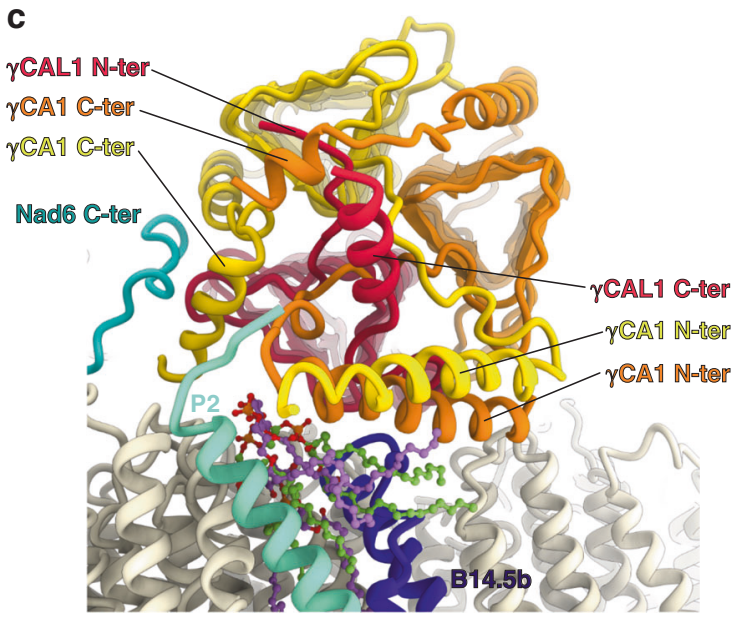

d

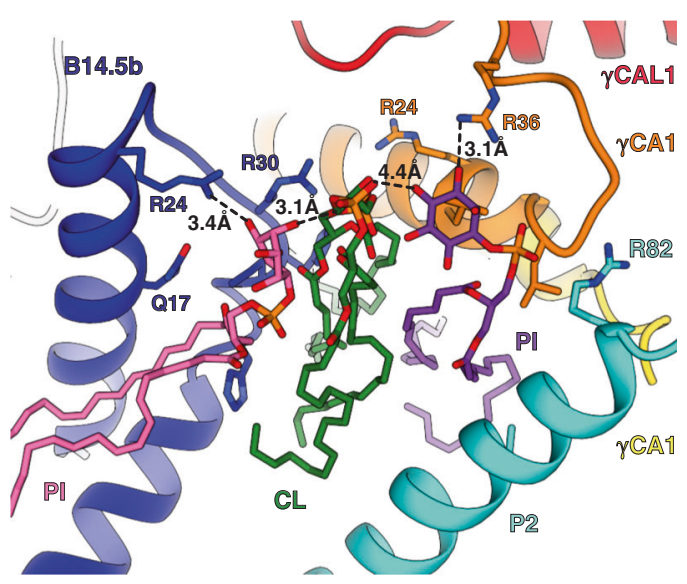

Fig. 2 Hetero-trimeric $\boldsymbol{\gamma}$-carbonic anhydrase of the plant mitochondrial complex I. a-c Front and back view of the carbonic anhydrase showing individual subunits and contacting proteins. Active and inactive sites are shown in $\mathbf{a}$. $\mathbf{b}$ Close up of the $\gamma \mathrm{CA} 1 / \gamma \mathrm{CA} 1$ only conserved active site, showing the coordinate zinc by the three histidine residues between the two $\gamma C A 1$ subunits. Sequence alignment of $\gamma C A$ and $\gamma C A L$ highlight the catalytic histidines. Surrounding residues, Tyr207, Gln101, Arg86, are also shown and might be involved in $\mathrm{HCO}_{3}{ }^{-}$coordination. c Back view of the carbonic anhydrase, highlighting the $\mathrm{N}$ and $\mathrm{C}$-ter extensions of the $\gamma \mathrm{CA}$ and $\gamma \mathrm{CAL}$, as well as the proteins allowing to anchor the whole domain to the membrane arm. $\mathbf{d}$ The lipid block composed of one central cardiolipin and two phosphatidylinositol molecules is shown in a close-up view, with surrounding residues. The lipids are sandwiched between the carbonic anhydrase domain, the plant-specific protein P2, Nad2, and B14.5. Both inositol head groups contribute to coordinate the cardiolipin. $\mathrm{CL}$ stands for cardiolipin and PI for phosphatidylinositol.

is undetectable, which hinted its role as a plant specific assembly factor ${ }^{13,27}$. Using focused classification and refinement, we were able to derive an intermediate-resolution reconstruction on the additional density corresponding to GLDH which was sufficient to attribute the density to the protein. Importantly, the complex I binding domain of GLDH is resolved at high resolution (3.8), thus validating our assignment (Fig. 3b-d). In this assembly intermediate, GLDH interacts with $\mathrm{B} 14.5 \mathrm{~b}$ - that is slightly displaced compared to the mature complex-and the $15 \mathrm{kDa}$ subunit. Moreover the whole carbonic anhydrase is slightly shifted toward the missing $\mathrm{P}_{\mathrm{D}}$ module (Fig. $3 \mathrm{c}$ ). We also successfully identified the N-terminal tail of GLDH bound to the maturing complex I, between $\mathrm{B} 14.5 \mathrm{~b}$ and $15 \mathrm{kDa}$ preventing the plant specific protein $\mathrm{P} 1$ from docking to the $\mathrm{P}_{\mathrm{P}}$ module. $\mathrm{P} 1$ appears to functionally replace the NDUFB5 subunit, which is absent in plants $^{23}$ (Supplementary Fig. 5). In the GLDH context the P1 path is blocked in the assembly intermediate due to B14.5b movement and N-ter tail of GLDH (Fig. 3). Thus, it appears that GLDH prevents the binding of $\mathrm{P} 1$ to the $\mathrm{P}_{\mathrm{P}}$ module, until its release, which will then allow association with the $\mathrm{P}_{\mathrm{D}}$ module. The clear mechanism of action of GLDH in the context of the assembly intermediate is however unclear. It was previously shown that GLDH can perform its original enzymatic activity, however this activity is not required from complex I formation, its functions are thus not linked ${ }^{13}$. Hence, in plants, GLDH might act as a stabilization factor of complex I assembly intermediates that would be released just before the final step of $\mathrm{P}_{\mathrm{D}}$ module docking to complex $\mathrm{I}^{*}$. It is unlikely that $\mathrm{P} 1$ alone could remove GLDH, thus this process would probably involve unknown additional trans-acting factor(s). Similarly, in yeast the assembly factor NDUFAF2 is released from the assembly intermediate by the joint action of three accessory subunits ${ }^{8}$.

In conclusion, our high-resolution cryo-EM structures of the plant mitochondrial complex I, reveals plant-specific and ancestral features of this respiratory complex (Fig. 4). Indeed, $\gamma \mathrm{CA}$ was identified as a complex I component in all Viridiplantae, including algae of the Chlorophyceae class ${ }^{11,28-31}$, as well as in Euglena gracilis, a photosynthetic protozoan related to trypanosomes $^{32}$ and possibly Acanthamoeba castellanii, a protozoan ameboid $^{33}$ and in non-photosynthetic organisms (e.g., Tetrahymena and Reclinomonas) ${ }^{33}$ (Fig. 4). Given the wide presence of the $\gamma \mathrm{CA}$ in eukaryotes-members of the $\gamma \mathrm{CA}$ are found in all 


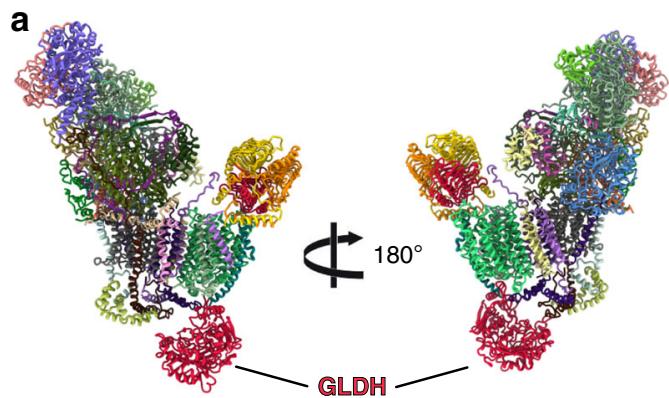

b
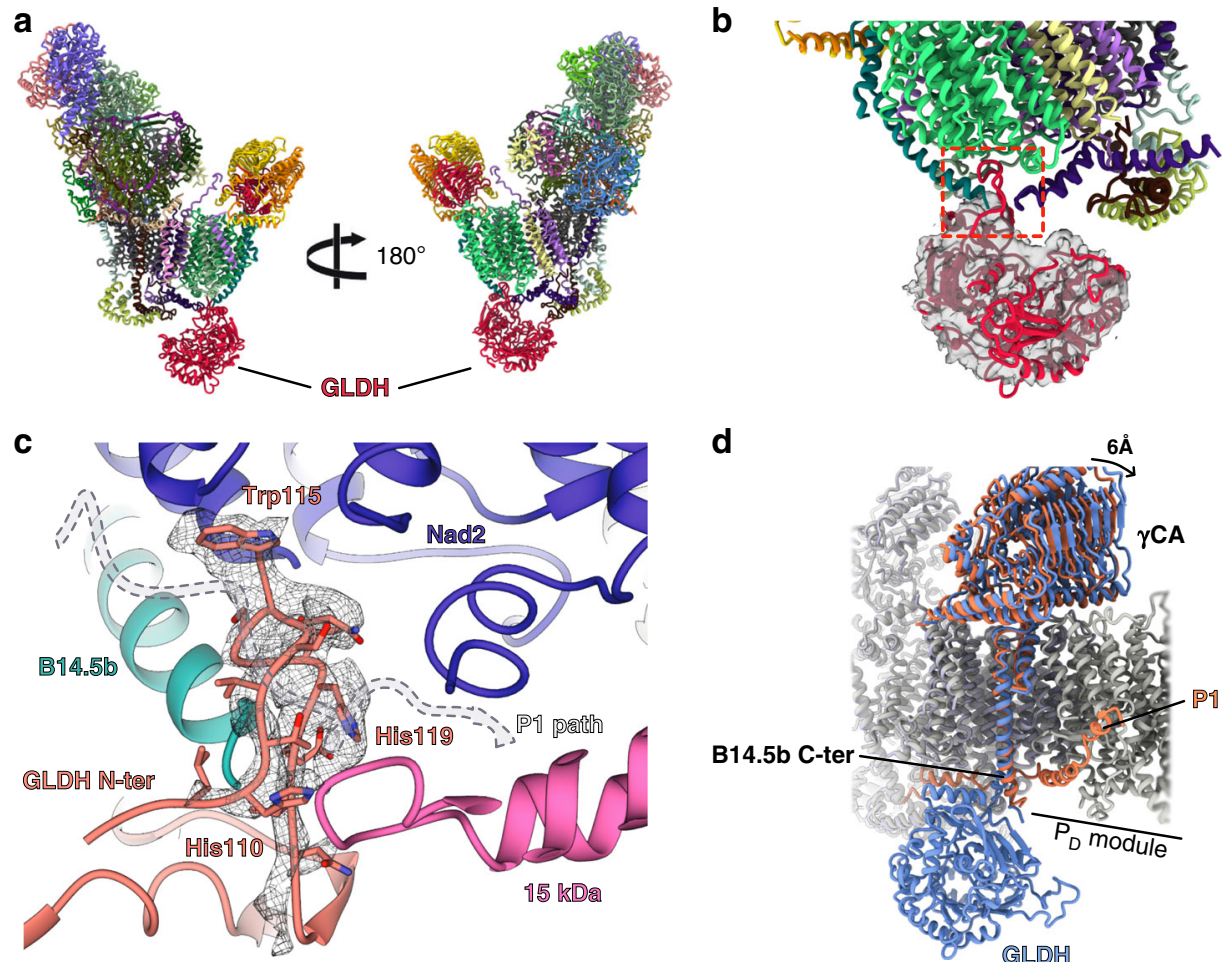

Fig. 3 GLDH is a plant-specific assembly factor of mitochondrial complex $\mathbf{I}$. a Atomic model of the assembly intermediate, the whole $P_{D}$ module is missing and GLDH contacts the $P_{p}$ module on the intermembrane side. $\mathbf{b}$ GLDH is shown in cut-out and filtered density. The interaction point is highlighted and a zoomed view is shown in c. $\mathbf{c}$ Focused view of the interaction point of GLDH with the complex. The N-terminal part of GLDH was identified thanks to specific aromatic residues and is shown in its density. The $\mathrm{N}$-ter part of GLDH, as well as the deviation of B14.5b block P1 path which is shown in dashed lines. d Overall movement in the assembly intermediate compared to the mature complex, B14.5b C-terminal part is deviated by $4 \AA$ and the whole $\gamma C A$ domain is shifted by $6 \AA$ toward the missing $P_{D}$ module, GLDH and absence of the P1 protein are also highlighted. Coral correspond to mature complex I and blue to assembly intermediate.

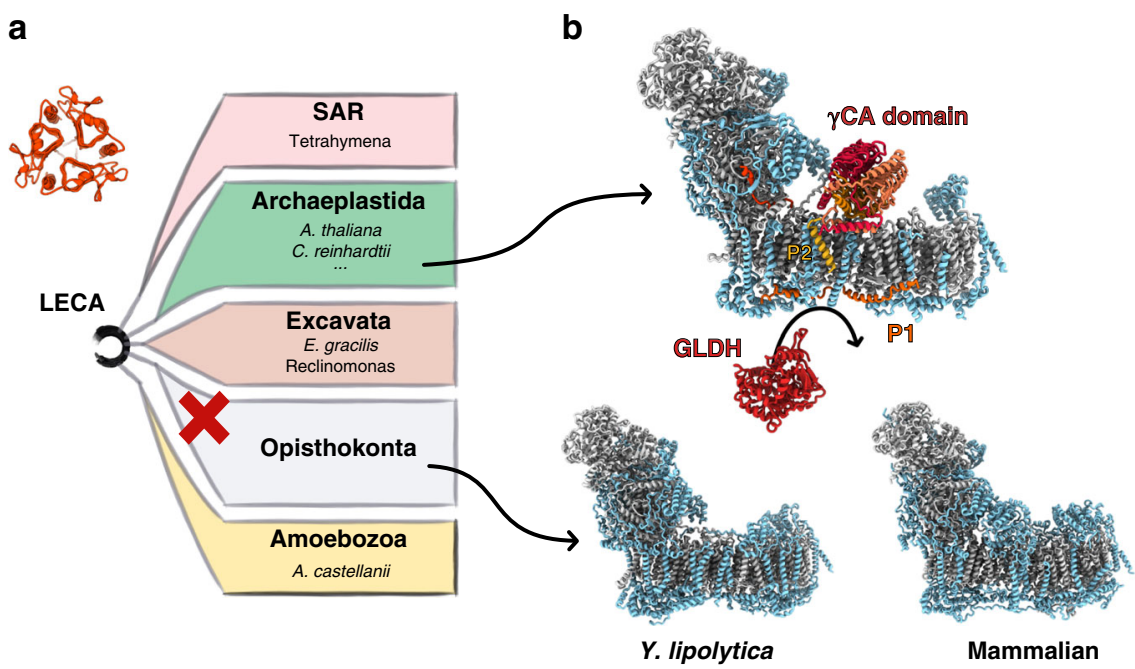

Fig. 4 Plant mitochondrial complex I specific subunits. a Schematic representation of the five major eukaryote lineages. Organisms where $\gamma$-carbonic anhydrase is proposed to be part of mitochondrial complex I are listed next to their respective lineage. The red cross indicate the loss of mitochondrial $\gamma$ carbonic anhydrase in opisthokonta. b Comparison of mitochondrial complex I structures. Core subunits are shown in light gray and supernumary subunits in light blue. Plant specific proteins are highlighted in red-orange colors. Y. lipolytica (PDB:6RFR) and mammalian (PDB:6G2J) complex I do not have the $\gamma^{-}$ carbonic anhydrase domain.

eukaryotic lineages except opisthokonts-we present for the first time the structure of a mitochondrial complex I featuring this ancestral component. Moreover, we describe a yet unobserved plant-specific assembly intermediate, shedding light into the respiratory complex I maturation.

\section{Methods}

Mitochondrial complex I purification. Cauliflower (Brassica oleracea var. botrytis) mitochondria were purified as previously described ${ }^{12}$. Quickly, fresh cauliflower inflorescence tissue was blended in extraction buffer containing $0.3 \mathrm{M}$ mannitol, 30 $\mathrm{mM}$ sodium pyrophosphate $\left(10 . \mathrm{H}_{2} \mathrm{O}\right), 0.5 \% \mathrm{BSA}, 0.8 \%(\mathrm{w} / \mathrm{v})$ polyvinylpyrrolidone$25,2 \mathrm{mM}$ beta-mercaptoethanol, $1 \mathrm{mM}$ EDTA, $20 \mathrm{mM}$ ascorbate and $5 \mathrm{mM}$ 
cysteine, $\mathrm{pH}$ 7.5. Lysate was filtered and clarified by centrifugation at $1500 \times g, 10$ min at $4{ }^{\circ} \mathrm{C}$. Supernatant was kept and centrifuged at $18,000 \times g, 15 \mathrm{~min}$ at $4{ }^{\circ} \mathrm{C}$. Organelle pellet was re-suspended in wash buffer $(0.3 \mathrm{M}$ mannitol and $10 \mathrm{mM}$ phosphate buffer, $1 \mathrm{mM}$ EDTA, pH 7.5) and the precedent centrifugations were repeated once. The resulting organelle pellet was re-suspended in wash buffer and loaded on a single-step $30 \%$ Percoll gradient (in wash buffer without EDTA) and run for $1 \mathrm{~h} 30$ at $40,000 \times g$. Mitochondria were retrieved, washed two times and flash frozen in liquid nitrogen.

For complex I purification, mitochondria were re-suspended in Lysis buffer $(20$ mM HEPES-KOH, pH 7.6, 100 mM KCl, 1 mM DTT, 2\% n- $\beta$-DDM, 1 mM EDTA, supplemented with proteases inhibitors (Complete)) incubated for $15 \mathrm{~min}$ in $4^{\circ} \mathrm{C}$. Lysate was clarified by centrifugation at $30,000 \times g, 20 \mathrm{~min}$ at $4{ }^{\circ} \mathrm{C}$. The supernatant was loaded on a $10-50 \%$ sucrose gradient buffer (20 mM HEPES-KOH, pH 7.6, 50 $\mathrm{mM} \mathrm{KCl}, 1 \mathrm{mM}$ DTT, $0.2 \% \mathrm{n}-\beta$-DDM, $1 \mathrm{mM}$ EDTA, supplemented with proteases inhibitors (Complete)) and run for $16 \mathrm{~h}$ at $75,000 \times g$. The fraction corresponding to complex I was collected, pelleted and re-suspended in final resuspension buffer (same as sucrose gradient buffer without sucrose and only $0.1 \% \mathrm{n}-\beta-\mathrm{DDM}$ ).

Grid preparation. Four microliter of the samples at a concentration of $1 \mu \mathrm{g} / \mu \mathrm{l} \mathrm{was}$ applied onto Quantifoil R2/2 300-mesh holey carbon grid, coated with thin homemade continuous carbon film and glow-discharged ( $3 \mathrm{~mA}$ for $20 \mathrm{~s}$ ). The sample was incubated on the grid for $30 \mathrm{~s}$ and then blotted with filter paper for $2.5 \mathrm{~s}$ in a temperature and humidity controlled Vitrobot Mark IV $\left(\mathrm{T}=4^{\circ} \mathrm{C}\right.$, humidity $100 \%$, blot force 5) followed by vitrification in liquid ethane.

Single particle cryo-electron microscopy data collection. Data collection was performed on a Talos Arctica instrument (Thermofisher Company) at $200 \mathrm{kV}$ using the SerialEM software for automated data acquisition. Data were collected at a nominal underfocus of -0.5 to $-2.5 \mu \mathrm{m}$ at a magnification of $\times 36,000$ yielding a pixel size of $1.11 \AA$ A. Micrographs were recorded as movie stack on a K2 direct electron detector (GATAN Company), each movie stack were fractionated into 65 frames for a total exposure of $6.5 \mathrm{~s}$ corresponding to an electron dose of $45 \mathrm{e} / \AA 2$.

Electron microscopy image processing. Drift and gain correction and dose weighting were performed using MotionCorr $2^{34}$. A dose weighted average image of the whole stack was used to determine the contrast transfer function with the software Gctf ${ }^{35}$. The following process has been achieved using RELION 3. 14 Particles were picked using the general model of crYOLO $1.5^{36}$. Output box files from crYOLO were imported in RELION and particles were extracted. After reference-free 2D classification, 650,844 particles were extracted with a box size of 360 pixels and binned threefold, resulting in a 120 pixels box size and used for 3D classification into 6 classes (Supplementary Fig. 1). CryoSPARC ${ }^{37}$ generated abinitio cryo-EM map, low-pass filtered to $30 \AA$, was used as an initial reference for 3D classification. One subclass depicting high-resolution features have been selected for the mature complex I refinement with 65,018 particles. After Bayesian polishing a focused refinement has been performed using mask excluding the $P_{D}$ module yielding, respectively, 3.7 and $3.47 \AA$ resolution. A second subclass containing the maturation factor GLDH composed of 155,644 particles was selected for further focused 3D classification, using a loose soft mask on the GLDH area. The 36,513 particles of GLDH enriched subclass were extracted for refinement and reached $3.8 \AA$ A . Determination of the local resolution of the final density map was performed using ResMap ${ }^{38}$.

Structure building and model refinement. The atomic model of the plant mitochondrial complex I was built into the high-resolution maps using Coot, Phenix, and Chimera. Atomic models from Y.lipolytica (6RFR) and M.musculus (6G2J) were used as starting points for protein identification and modelisation. Similarly to Waltz \& Soufari $2019^{12}$, as the genome of cauliflower is not sequenced, and the closest fully sequenced member of the family (Brassica oleracea subsp. oleracea) is poorly annotated, to facilitate comprehension and analysis we positioned Arabidopsis proteins in the cauliflower map. Still, protein sequence identities between members of the Brassicaceae family are higher than $90 \%$, thus facilitating proteomics identification of cauliflower proteins. The online SWISSMODEL service was used to generate initial models for bacterial and mitochondria conserved r-proteins. Models were then rigid body fitted to the density in Chimera $^{39}$ and all subsequent modeling was done in $\operatorname{Coot}^{40}$. Extensions were built has polyalanine and mutated to the adequate sequences. A combination of regularization and real-space refine was performed in Coot for each proteins. The global atomic model was then subjected to real space refinement cycles using phenix. real_space_refine PHENIX ${ }^{41}$ function, during which protein secondary structure, Ramachandran and side chain rotamer restraints. Several rounds of refinement (manual in Coot and automated using the phenix.real_space_refine) were performed to obtain the final models, which were validated using the built-in validation tool of PHENIX, based on MolProbity. For the assembly intermediate, the model was built with the mature complex as template. Refinement and validation statistics were summarized in Supplementary Table 2.

Proteomic and statistical analyses of mitochondrial complex I composition Mass spectrometry analyses of the complex I fractions were performed at the
Strasbourg-Esplanade proteomic platform and performed as previously ${ }^{42}$. In brief, proteins were trypsin digested, mass spectrometry analyses and quantitative proteomics were carried out by nanoLC-ESI-MS/MS analysis on a QExactive+ (Thermo) mass spectrometer. Data were searched against the TAIR A. thaliana database with a target-decoy strategy (release TAIRv10, 27281 forward protein sequences), and home-made Brassica oleracea database extracted from UniProtKB (Swissprot+TrEMBL) including Brassica sub-taxonomy (release 2017_10, 59050 forward protein sequences). Proteins were validated respecting FDR $<1 \%$ (False Discovery Rate) and quantitative label-free analysis was performed through inhouse bioinformatics pipelines.

Figure preparation. Figures featuring cryo-EM densities, as well as atomic models were visualized with UCSF ChimeraX ${ }^{43}$ and Chimera ${ }^{39}$.

Reporting summary. Further information on research design is available in the Nature Research Reporting Summary linked to this article.

\section{Data availability}

The cryo-EM maps of plant mitochondrial complex I have been deposited at the Electron Microscopy Data Bank (EMDB): EMD-11614 for the mature complex, EMD-11513 for the focused reconstruction, EMD-11615 for the assembly intermediate. The corresponding atomic models been deposited in the Protein Data Bank (PDB) under the accession 7A23 for the mature complex and 7A24 for the assembly intermediate. Mass spectrometric data have been deposited to the ProteomeXchange Consortium via the PRIDE partner repository with the dataset identifier PXD017847.

Received: 5 April 2020; Accepted: 9 September 2020; Published online: 15 October 2020

\section{References}

1. Sazanov, L. A. A giant molecular proton pump: structure and mechanism of respiratory complex I. Nat. Rev. Mol. Cell Biol. 16, 375-388 (2015).

2. Baradaran, R., Berrisford, J. M., Minhas, G. S. \& Sazanov, L. A. Crystal structure of the entire respiratory complex I. Nature 494, 443-448 (2013).

3. Gray, M. W. Mitochondrial evolution. Cold Spring Harb. Perspect. Biol. 4, a011403 (2012).

4. $\mathrm{Gu}, \mathrm{J}$. et al. The architecture of the mammalian respirasome. Nature 537, 639-643 (2016).

5. Agip, A.-N. A. et al. Cryo-EM structures of complex I from mouse heart mitochondria in two biochemically defined states. Nat. Struct. Mol. Biol. 25 548-556 (2018).

6. Fiedorczuk, K. et al. Atomic structure of the entire mammalian mitochondrial complex I. Nature 538, 406-410 (2016).

7. Zhu, J., Vinothkumar, K. R. \& Hirst, J. Structure of mammalian respiratory complex I. Nature 536, 354-358 (2016).

8. Parey, K. et al. High-resolution cryo-EM structures of respiratory complex I mechanism, assembly, and disease. Sci. Adv. 5, eaax9484 (2019).

9. Parey, K. et al. Cryo-EM structure of respiratory complex I at work. Elife 7, e39213 (2018).

10. Eubel, H., Heinemeyer, J. \& Braun, H.-P. Identification and characterization of respirasomes in potato mitochondria. Plant Physiol. 134, 1450-1459 (2004).

11. Dudkina, N. V., Eubel, H., Keegstra, W., Boekema, E. J. \& Braun, H.-P. Structure of a mitochondrial supercomplex formed by respiratory-chain complexes I and III. Proc. Natl Acad. Sci. USA 102, 3225-3229 (2005).

12. Waltz, F., Soufari, H., Bochler, A., Giegé, P. \& Hashem, Y. Cryo-EM structure of the RNA-rich plant mitochondrial ribosome. Nat. Plants $\mathbf{6}$, 377-383 (2020)

13. Schimmeyer, J., Bock, R. \& Meyer, E. H. 1-Galactono-1,4-lactone dehydrogenase is an assembly factor of the membrane arm of mitochondrial complex I in Arabidopsis. Plant Mol. Biol. 90, 117-126 (2016).

14. Zivanov, J. et al. New tools for automated high-resolution cryo-EM structure determination in RELION-3. Elife 7, e42166 (2018).

15. Letts, J. A., Fiedorczuk, K. \& Sazanov, L. A. The architecture of respiratory supercomplexes. Nature 537, 644-648 (2016).

16. Momayyezi, M., McKown, A. D., Bell, S. C. S. \& Guy, R. D. Emerging roles for carbonic anhydrase in mesophyll conductance and photosynthesis. Plant $J$. tpj.14638 https://doi.org/10.1111/tpj.14638 (2020).

17. Tripp, B. C., Bell, C. B., Cruz, F., Krebs, C. \& Ferry, J. G. A role for iron in an ancient carbonic anhydrase. J. Biol. Chem. 279, 6683-6687 (2004).

18. Alber, B. E. et al. Kinetic and spectroscopic characterization of the gammacarbonic anhydrase from the methanoarchaeon methanosarcina thermophila. Biochemistry 38, 13119-13128 (1999).

19. Parisi, G. et al. Gamma carbonic anhydrases in plant mitochondria. Plant Mol. Biol. 55, 193-207 (2004). 
20. Fromm, S., Senkler, J., Zabaleta, E., Peterhänsel, C. \& Braun, H.-P. The carbonic anhydrase domain of plant mitochondrial complex I. Physiol. Plant. 157, 289-296 (2016).

21. Perales, M. et al. Gamma carbonic anhydrase like complex interact with plant mitochondrial complex I. Plant Mol. Biol. 56, 947-957 (2004).

22. Cot, S. S. W., So, A. K. C. \& Espie, G. S. A multiprotein bicarbonate dehydration complex essential to carboxysome function in cyanobacteria. $J$. Bacteriol. 190, 936-945 (2008).

23. Ligas, J., Pineau, E., Bock, R., Huynen, M. A. \& Meyer, E. H. The assembly pathway of complex I in Arabidopsis thaliana. Plant J. 97, 447-459 (2019).

24. Schuller, J. M. et al. Redox-coupled proton pumping drives carbon concentration in the photosynthetic complex I. Nat. Commun. 11, 494 (2020).

25. Jussupow, A., Di Luca, A. \& Kaila, V. R. I. How cardiolipin modulates the dynamics of respiratory complex I. Sci. Adv. 5, eaav1850 (2019).

26. Millar, A. H. et al. Control of ascorbate synthesis by respiration and its implications for stress responses. Plant Physiol. 133, 443-447 (2003)

27. Pineau, B., Layoune, O., Danon, A. \& De Paepe, R. 1-Galactono-1,4-lactone dehydrogenase is required for the accumulation of plant respiratory complex I. J. Biol. Chem. 283, 32500-32505 (2008).

28. Davies, K. M., Blum, T. B. \& Kühlbrandt, W. Conserved in situ arrangement of complex I and III2 in mitochondrial respiratory chain supercomplexes of mammals, yeast, and plants. Proc. Natl Acad. Sci. USA 115, 3024-3029 (2018).

29. Subrahmanian, N., Remacle, C. \& Hamel, P. P. Plant mitochondrial Complex I composition and assembly: a review. Biochim. Biophys. Acta Bioenerg. 1857, 1001-1014 (2016).

30. Sunderhaus, S. et al. Carbonic anhydrase subunits form a matrix-exposed domain attached to the membrane arm of mitochondrial complex I in plants. J. Biol. Chem. 281, 6482-6488 (2006).

31. Klodmann, J., Sunderhaus, S., Nimtz, M., Jansch, L. \& Braun, H. P. Internal Architecture of Mitochondrial Complex I from Arabidopsis thaliana. Plant Cell 22, 797-810 (2010).

32. Miranda-Astudillo, H. V. et al. The atypical subunit composition of respiratory complexes I and IV is associated with original extra structural domains in Euglena gracilis. Sci. Rep. 8, 9698 (2018).

33. Gawryluk, R. M. R. \& Gray, M. W. Evidence for an early evolutionary emergence of gamma-type carbonic anhydrases as components of mitochondrial respiratory complex I. BMC Evol. Biol. 10, 176 (2010).

34. Zheng, S. Q. et al. MotionCor2: anisotropic correction of beam-induced motion for improved cryo-electron microscopy. Nat. Methods 14, 331-332 (2017).

35. Zhang, K. Gctf: Real-time CTF determination and correction. J. Struct. Biol. 193, 1-12 (2016).

36. Wagner, T. et al. SPHIRE-crYOLO is a fast and accurate fully automated particle picker for cryo-EM. Commun. Biol. 2, 218 (2019).

37. Punjani, A., Rubinstein, J. L., Fleet, D. J. \& Brubaker, M. A. cryoSPARC algorithms for rapid unsupervised cryo-EM structure determination. Nat. Methods 14, 290-296 (2017).

38. Kucukelbir, A., Sigworth, F. J. \& Tagare, H. D. Quantifying the local resolution of cryo-EM density maps. Nat. Methods 11, 63-65 (2014).

39. Pettersen, E. F. et al. UCSF Chimera-a visualization system for exploratory research and analysis. J. Comput. Chem. 25, 1605-1612 (2004).

40. Emsley, P., Lohkamp, B., Scott, W. G. \& Cowtan, K. Features and development of Coot. Acta Crystallogr. Sect. D. Biol. Crystallogr. 66, 486-501 (2010).

41. Liebschner, D. et al. Macromolecular structure determination using X-rays, neutrons and electrons: recent developments in Phenix. Acta Crystallogr. Sect. D. Struct. Biol. 75, 861-877 (2019).

42. Waltz, F. et al. Small is big in Arabidopsis mitochondrial ribosome. Nat. Plants 5, 106-117 (2019).
43. Goddard, T. D. et al. UCSF ChimeraX: meeting modern challenges in visualization and analysis. Protein Sci. 27, 14-25 (2018).

\section{Acknowledgements}

This work has benefitted from the facilities and expertize of the Biophysical and Structural Chemistry platform (BPCS) at IECB, CNRS UMS3033, Inserm US001, University of Bordeaux. We thank A. Bezault for assistance with the Talos Arctica electron microscope. We thank, J. Chicher and P. Hamman of the Strasbourg Espanade proteomic analysis for the proteomic analysis. This work was supported by a European Research Council Starting Grant (TransTryp ID:759120) and a Agence Nationale de la Recherche (ANR) grant [MITRA, ANR-16-CE11-0024-02] to Y.H.

\section{Author contributions}

F.W., H.S., and Y.H. designed and coordinated the experiments. F.W. purified the mitochondria and mitochondrial complex I. H.S. and C.P. prepared grids. H.S. acquired the cryo-EM data and processed the cryo-EM results with F.W. H.S. and F.W. built the atomic models. L.K. performed the mass-spectrometry experiments. F.W., H.S. and Y.H. interpreted the structure. F.W., H.S., C.P., and Y.H. wrote and edited the manuscript.

\section{Competing interests}

The authors declare no competing interests.

\section{Additional information}

Supplementary information is available for this paper at https://doi.org/10.1038/s41467020-18814-w.

Correspondence and requests for materials should be addressed to F.W. or Y.H

Peer review information Nature Communications thanks Friedrich Förster, Kutti Vinothkumar and Eduardo Zabaleta for their contribution to the peer review of this work. Peer review reports are available.

Reprints and permission information is available at http://www.nature.com/reprints

Publisher's note Springer Nature remains neutral with regard to jurisdictional claims in published maps and institutional affiliations.

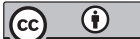

Open Access This article is licensed under a Creative Commons Attribution 4.0 International License, which permits use, sharing, adaptation, distribution and reproduction in any medium or format, as long as you give appropriate credit to the original author(s) and the source, provide a link to the Creative Commons license, and indicate if changes were made. The images or other third party material in this article are included in the article's Creative Commons license, unless indicated otherwise in a credit line to the material. If material is not included in the article's Creative Commons license and your intended use is not permitted by statutory regulation or exceeds the permitted use, you will need to obtain permission directly from the copyright holder. To view a copy of this license, visit http://creativecommons.org/ licenses/by/4.0/.

(C) The Author(s) 2020 